\title{
The meaning of community involvement in health: the perspective of primary health care communities
}

\author{
GG Mchunu, RN, M Cur \\ University of KwaZulu Natal
}

NS Gwele, RN, PhD

Durban Institute of Technology *(Note during the course of this study: University of KwaZulu-Natal)

\section{Keywords:}

Community involvement: health

\section{Abstract: Curationis 28(2): $30-37$}

The goal of this study was to establish the understanding and appreciation of the essence of PHC principles in the two Primary Health Care (PHC) communities. The PHC communities in this study referred to the people who were involved in the operation of the phenomenon, that is health professionals working in the health care centers and the communities served by these health care centers. It was hoped that the study would enhance the understanding of the importance of community involvement in health $(\mathrm{CIH})$ in health care delivery, for both community members and health professionals.

A case study method was used to conduct the study. Two community health centers in the Ethekwini health district, in Kwa Zulu Natal, were studied. One health center was urban based, the other was rural based. A sample of 31 participants participated in the study. The sample comprised of 8 registered nurses, 2 enrolled nurses, 13 community members and 8 community health workers. Data was collected using individual interviews and focus groups, and was guided by the case study protocol.

The findings of the study revealed that in both communities, participants had different albeit complementary, understanding of the term 'Community Involvement in Health' (CIH). Essentially, for these participants, $\mathrm{CIH}$ meant collaboration, co-operation and involvement in decision-making.

\section{Background to the problem}

One of the primary health care (PHC) principles that were identified at the Alma Ata conference held in 1978 was the principle of community participation or community involvement in health (WHO, 1978: 2). Presently, very little is known as to the extent of community involvement in health $(\mathrm{CIH})$ in South Africa. Despite the fact that the government has advocated community involvement as an important attribute in the delivery of health care. In developing the National Health Plan, the African National Congress (ANC) government based the plan on the Alma- Ata principles of comprehensive primary health care (ANC, 1994: 20). The government's intention to provide primary health care to all the people was demonstrated in the National Health Service Delivery Plan. This plan stated that an affordable, comprehensive health service should be developed, in which community members would have the power to decide on health issues (ANC, 1994: 21). In The White Paper for Transformation of the Health System in South Africa (Department of Health, 1997: 14) the objectives for restructuring the health system were set. One of these objectives was to foster community participation across the health sector by involving communities in various aspects of the planning and provision of health services (Department of Health, 1997: 16). In this document the Government indicates that community participation in health promotion and health service provision is an important component in the improvement of health 
care (Van Rensburg, 2004:131).

The findings of the survey of the national primary health facilities, conducted by Viljoen, Heunis, Janse van Rensburg, van Rensburg, Engelbrecht, Fourie, Steyn, and Matebesi (2000:82), showed that little progress had been made in facilitating community participation in PHC in South Africa in the two years since 1998. Viljoen et al. in this study further argue that even though KwaZulu Natal (KZN) was among the provinces where headway had been made, the change was described as limited.

Owing to the geographic structure and conditions of the communities in South Africa, and specifically in the KZN province, effective $\mathrm{CIH}$ might not always be easy to achieve. The nature of urban communities does not allow for the kind of interaction between community members and health professionals, which is needed for effective CIH. Urban communities are not as closely knit as rural communities are. The community members in urban communities tend to come together only in times of crisis and/ or need, rather than as a way of living. It would therefore seem that urban and rural communities present different challenges for $\mathrm{CIH}$, with a particular understanding of community involvement having been developed in each setting.

This study was aimed at enhancing the understanding of policy on $\mathrm{CIH}$, and the importance of $\mathrm{CIH}$ in health care delivery, for both community members and health professionals. The more recent study conducted for the health systems trust (HST) by Gwele and Makhanya (2001:1718) revealed that community involvement in health had taken many forms from mere establishment of clinic committees to intersectorial collaboration, focusing on more than health issues, by including community development as a whole. This range demonstrated the significance of community involvement in health care delivery and indicated a need to determine the understanding of community involvement by the PHC communities.

\section{Purpose and objectives of the study}

The purpose of this study was to explore and describe PHC communities, understanding and appreciation of the essence of $\mathrm{CIH}$ as a principle of PHC. The objectives were:

1. To describe urban and rural PHC communities' understanding of $\mathrm{CIH}$.
2. To determine similarities and differences between the rural and urban communities' understanding and practice of $\mathrm{CIH}$.

\section{Definition of terms}

Community: It is a group of people who share some type of bond. who interact with each other, and who function collectively regarding common concerns. The bond may take many forms, in that it can be shared ethnicity or culture or living in a specific geographic location or it can take the form of similar interests, goals, or occupations (Clark, 1996:6). In addition to this definition the ANC's definition of the term community will be adopted, that is, "to represent those people living in the geographical area served by a community health centre" (ANC, 1994:61).

Community involvement and/or participation: This refers to a shift in emphasis from external agencies supplying health services, to the people of a community becoming active participants in their own health care. This means that the community members become partners in health care by generating their own ideas; assessing their needs, by involvement in decisionmaking processes, planning, implementing, and even evaluating the care they receive (Dennill, King \& Swanepoel, 1999: 9). These two terms will be used interchangeably.

Primary Health Care (PHC) communities: In this study, this term refers to people who are involved in the operation of the phenomenon, that is, the health professionals working in the health care centre and the community served by the health centre.

Health professionals: These are the members of the health team, including the nurses (all categories) working in community health centres.

Rural community: Geographically, this term refers to areas that are remote and isolated. Rural communities are not homogeneous, but the following characteristics for rural communities will be assumed, namely, (a) sparse population, (b) low family income, (c) unemployment, (d) poor schools, and (e) inadequate or inaccessible health care systems (Deloughery, 1998:359).

Urban community: In this study, this term refers to communities situated in the inner city, in which a large number of people live and work in close proximity. The assumption will be based on Mann's (1983: 409) definition that in urban communities relationships are impersonal and superficial and segmental. Also, that the population is more heterogeneous owing to greater mobility of the people.

\section{Literature survey}

In the literature review, the following key concepts were explored:

(a) the community, (b) primary health care (PHC), and (c) community involvement (participation). Relevant research articles on community participation in health problems and on measuring community participation were reviewed. In this article the focus will be on community participation.

\section{Community Involvement and/or Participation in Health}

Community participation plays an important role in rendering effective $\mathrm{PHC}$ in the community. The community can participate in many ways and at every stage of PHC. According to the WHO (1978: 51,) the community can be involved in the assessment of the situation, the definition of problems and the setting of priorities, and can then help to plan PHC activities and co-operate fully when these activities are carried out. It is important that the community is willing to participate. The health system will then be involved in explaining and providing information where necessary.

\section{Conceptualisations of Community involvement and/or Participation In Health}

The term "community involvement" and/ or "participation in health" is an outgrowth of various attempts at determining both the substance and the process of community involvement in the provision of health care. It first emerged or became popularised in health related literature following the Alma Alta conference in 1978. At this conference, the WHO identified community participation as one of the principles of PHC. The organization defined community participation as "the process by which the individual and the families assume responsibility for their own health and welfare and for that of the community, and develop the capacity to contribute to their community's development" (WHO, 1978: 50). Various 
Table 1: Illustrating three forms of community involvement

\begin{tabular}{|l|l|l|}
\hline WHO(1985) & Chimera- Dan (1996) & Kahssay and Oakley (1999) \\
\hline Contribution & Program instrument & Collaboration \\
\hline Organization & Partnership & Specific targeting of project benefit \\
\hline Empowerment & Empowerment & Empowerment \\
\hline
\end{tabular}

authors (Chimera-Dan, 1996: 13; Kahssay \& Oakley 1999: 5; WHO 1985: 4) have conceptualised the term community participation as consisting of at least three forms. Table 1 depicts the three levels of community participation/ involvement as conceptualised by these authors.

In 1985 the WHO study group maintained that community participation could be seen in three forms, that is, as (a) contribution, (b) organization and (c) empowerment. This group further elaborated on these concepts as follows:

1. Contributive participation: occurs when the community participates in predetermined projects and programs through contributions of labour, cash or material.

2. Organization participation: involves the creation of the appropriate structures to facilitate participation.

3. Empowering participation: involves groups or communities, particularly those that are poor or marginalized, developing the power to make real choices concerning health care services, through having an effective say in or having control over these programs.

According to Rifkin, Muller and Bichmann (1988: 933), however, the WHO prefers the term community involvement' to the term 'community participation' because, according to the WHO, the term "community involvement" implies active participation rather than passive engagement in health activities.

A similar view of community participation and/or involvement was conceived by Chimera-Dan (1996:13), who identified three forms of community involvement. These were (a) a program instrument (b) partnership, and (c) community empowerment. These forms of community involvement were described as follows:

1. A program instrument: The community is used to advance the objectives of the program, and to improve efficiency, effectiveness and cost recovery of the project.
2. Partnership: This is formed with the local authority, arriving at a compromise between the community and the health authorities to organise self-help programs, respect for the individual and willingness by the authorities to cooperate.

3. Community empowerment: This refers to a means of prompting self-reliance and self-determination at both individual and community level.

Kahssay and Oakley (1999: 8) differentiate between the concepts 'community participation' and 'community involvement' in health. For these authors, community involvement differs from community participation in that the former is not just a mechanism to lend support to externally led health development programs. Community involvement is described as "a strategy, which systematically promotes community participation and supports and strengthens it in order to provide better health for the majority of people"(Kahssay \& Oakley, 1999: 8). They argue that $\mathrm{CIH}$ works as an umbrella that involves community participation. In other words, community participation is part of $\mathrm{CIH}$. For instance, they assert that $\mathrm{CIH}$ " increases the possibility that health programs and projects will be appropriate and successful in meeting the health needs defined by local people as opposed to those defined by health services"(Kahssay \& Oakley, 1999:9).

Woelk (1992: 420) cites Rifkin's definition of community participation, which does not differ in essence from what Kahssay and Oakley see as community involvement in health. Rifkin defines community participation as "a social process whereby a specific group with shared needs, living in a defined geographic area, pursues mechanisms to meet those needs" (Woelk, 1992: 420). Rifkin (1990) further defined community involvement in health as:

"a process by" which a partnership is established between the government and local communities in the planning, implementation and utilization of health activities in order to benefit from increased local self-reliance and social control over infrastructure and technology of PHC" (cited in Kahssay \& Oakley, 1999:10).

It is therefore, apparent from the above statements that, in fact Rifkin does not really differentiate between the two concepts, at least, in so far as the nature of the communities' participation and/or involvement in health is concerned. For Kahssay and Oakley (1999:5), however, the two concepts are distinct and separate. These authors see community participation as a process that ensures the local people's co-operation or collaboration with externally induced development programs and projects and therefore, facilitating the effective implementation of such activities.

Kahssay and Oakley (1999:5) view community participation as:

1. Collaboration: Here, people voluntarily, or as a result of some incentive, agree to collaborate with an externally determined development project often by contributing their labour and other resources in return for some other expected benefits.

2. Specific targeting of project benefits: Benefits are targeted directly at previously excluded groups, for example, landless people, the poor and so on. The beneficiaries influence the direction and execution of the development project rather than merely receiving a share of the project benefits.

3. Empowerment: Previously excluded groups are empowered in order to increase access to and control over development resources. This process includes the development of skills and abilities to enable people to manage existing development delivery systems better and to have a say in whatever is done.

The preceding discussion of the conceptualisation of the term "community participation' by Chimera-Dan (1996), Kahssay and Oakley (1999) and the WHO study group (1985) shows that community participation, although it usually is externally introduced, can and does go beyond the level of 'welfare activities', to ensure empowerment of the community.

\section{Research methodology}

The understanding of community participation in rural and urban 
communities was explored and analysed based on the different community types. The case study protocol was used to guide the researcher and to keep her focused on the purpose of the study.

\section{Research Design}

A qualitative research approach using the case study design was chosen for this study. A multiple case study design was used where cases were selected from both rural and urban communities. Multiple case studies were used because the findings arising from two different cases are considerea more powerful that those from a single case, which can enhance the generalizability of findings (Yin, 2003:53). These two cases also represented two contrasting situations, and this was deemed good for comparison of findings.

\section{Population}

The population consisted of community health centres, health professionals in these centres, and the surrounding communities, in the different community settings in the Ethekwini health district.

\section{Sampling}

The setting was community health centres in two different types of communities, namely rural and urban communities. Two community health centres within Ethekwini health district were chosen as suitable sites for this study because the district ranges between extreme urban and extreme rural communities. This district, according to the classification by the KZN department of health, consists of areas such as Pinetown, Durban, Chatsworth, Umlazi, , Inanda and iNdwedwe (http:// www.kzn.gov.za, accessed on 04/02/ 2005). (At the time when this study was conducted these areas were classified under Ilembe health district). Two cases (PHC communities) were selected for participation in the study. These comprised one case from rural community and one case from an urban community.

Purposive sampling was used as a method of sampling the two communities. This method was selected because the researcher needed to obtain views from individuals with different community backgrounds (Burns \& Grove, 2001:376).

\section{Case Description}

In this study the case was a community health centre (clinic) in the chosen communities with all the community members utilising the clinic, health programs and community health workers as its embedded units of analysis. The context of the case was the chosen urban (case A) and rural (case B) communities within the Ethekwini health district.

\section{Case Selection}

Purposive sampling was used to select cases. The researcher identified two particular types of cases for in- depth investigation (Neuman, 1997: 206). These cases were selected purposively to ensure that the chosen cases were typical of the population required (Seaman, 1987:244). One case was selected to represent the rural community and the second case was selected to represent the urban community.

A sampling frame, which had the names of all the areas classified under this health district, was used to select the cases. From the sampling frame, the researcher identified one rural and one urban community. Each case was carefully selected to ensure that it predicted contrasting results (Yin, 2003: 47).

\section{Sampling of Participants}

The most appropriate case sampling strategy for qualitative research is nonprobability sampling, one example of which is theoretical sampling. Theoretical sampling was used in the selection of study participants. The initial group of participants were nurses and community members in the two selected PHC communities. Since this method of sampling was used, the researcher kept on including other groups of participants, other than the nurses and the community members as the need arose. This was done, because in theoretical sampling. the researcher can do what Glaser and Strauss (1967: 49) refer to as 'ongoing inclusion of groups' and 'selection of comparison groups', which can be done when the researcher needs to turn to certain groups or sub-groups for the next data collection. In this study the researcher added the community health workers (CHWs), as participants, as they were also involved in health matters in the community.

A total of 31 participants, representing both cases, participated in this study.

Case A (urban community): All in all 17 participants were interviewed. These included 5 registered nurses, 6 community members who lived in the areas surrounding the clinic and $6 \mathrm{CHWs}$. Of the 5 registered nurses interviewed, 3 were in charge of the specific health programmes. These programmes were HIV/ AIDS, training of health personnel and the coordination of $\mathrm{CHW}$ programme.

Case B (rural community): A total of 14 participants were interviewed. The health professionals interviewed consisted of 3 registered nurses, one of whom was the person in charge of the clinic and 2 enrolled nurses. From the community, 2 izinduna were interviewed and 5 active community members. The CHWs included one community health workers' coordinator, who is in charge of the $\mathrm{CHWs}$ who are attached to the clinic, and one of the 4 volunteer CHWs who are not attached to the clinic.

In case $\mathrm{A}$, where active community members were not clearly identified, the researcher used convenience sampling to include community members. People who happened to be at the clinic at that time were identified as possible participants (Burns \& Grove, 2001:374; Polit and Hungler, 1999:281). The researcher visited the clinic over the period of one week and explained the proposed research to the clients who were waiting in the waiting area. The interested community members volunteered to participate, but could only be included if they were residents of this community and not just visiting.

In case B, community members were sampled using snowball sampling. This seemed to be the most appropriate method as the researcher was looking for people with specific traits (Polit and Hungler, 1999:281), namely people who had been utilizing the same clinic for a period of over 5 years. The criterion for selecting active community members was through identification of these individuals by a variety of sources. These included the nursing staff, community health workers (such as the AIDS co-ordinators, the community health workers' trainers and facilitators). The researcher also included those community members identified by community leaders and other informants (such as izinduna and other respected community members).

\section{Ethical considerations}

Permission to conduct the study was requested from the department of health in $\mathrm{KZN}$. Authorities from the different institutions concerned, namely, the various community health centres, were also approached for consent to conduct a study. Community leaders were also 
approached for their consent. All participants were asked for either a written or verbal informed consent and were given a choice to refuse to participate. They were informed that they were free to discontinue at any time of the study. Confidentiality was to be maintained at all times. The researcher asked for permission to record all interviews, including the focus groups. Participants were assured that no physical risks were involved in this study.

\section{Data collection}

Data collection in this study was guided by the case study protocol. According to Yin (2003: 67) a case study protocol is essential if one is doing a multiple case study.

This case study protocol contained the following:

- A short description of each case, in terms of its demography, services offered and present activities.

- An interview schedule with one research question to be asked, the main research question being "What is your understanding of community involvement in health?"

This research question was used in both the individual interviews and focus groups and the researcher had to probe to obtain more information.

The strategies used for data collection included face-to-face interviews in the form of focus group interviews and individual interviews. The interviews allowed the respondents to comment on widely defined issues and to feel free to expand on their experiences, as is characteristic of semi-structured interviews. Purposively selected focus group interviews were conducted where the informants were found as a group or for informants who were working together. This was to ensure that the groups were homogeneous, to facilitate open discussion (Burns \& Grove, 2001: 452). One focus group was conducted in each community. In case $A$ the focus group was conducted with the CHWs, whereas in case $\mathrm{B}$, the focus group was on community members. This difference was determined by the availability of participants.

\section{Data analysis}

Data collection and analysis was done simultaneously. The analysis of data was begun by using a template, in this case a case protocol. Template analytic techniques are more open-ended and include generation of themes, patterns and interrelationships in an interpretive rather than a statistical process (Crabtree \& Miller, 1992: 19). A case protocol, together with the research question, guided the analysis of data.

The researcher identified themes and patterns and did interpretational manual data analysis. Data was then segmented to meaningful units. The segments were coded and sorted into categories. Relationships among categories were then established. As described by Miles and Huberman, (1994:90) within case and cross case analysis was done to compare the findings in different settings. Information was put in different arrays, a matrix of categories was developed and evidence placed within such categories (Miles \& Huberman, 1994, in Yin, 2003: 111). These results were presented in tables.

\section{Trustworthiness}

To ensure richness and depth of data as well as to enhance credibility of this study, triangulation was implemented by utilizing multiple sources of data (Polit \& Hungler, 1999:428). For data triangulation multiple sources of data were used. These sources included using two cases, and having health professionals, community members and community health workers as study participants. For method triangulation, different methods of data collection were used and these included focus group interviews and individual interviews. Peer examination was achieved by discussing the findings with a colleague who is an experienced and credible researcher. Focus groups were also conducted for data verification and member checks.

\section{Results and discussion Understanding of $\mathrm{ClH}$ by the community members}

Perusal of Table 2 reveals that the term $\mathrm{CIH}$ meant various things to the rural and to the urban communities. From the data analysis it emerged that the urban community understood $\mathrm{CIH}$ as mutual assistance and organized participation. Mutual assistance referred specifically to community members assisting each other in times of need and assisting the health centre when the need arose. The following excerpts from the urban interviewees demonstrate these observations:

"It means working together as a community, helping people like those who have problems, if they are hungry or if they need food it means giving them food". (Urban community)

"It is about helping each other, the clinic helping us and us helping the clinic. It means having committees that stand for health matters in the community". (Urban community)

For the rural community, however, $\mathrm{CIH}$ meant more than mutual assistance. It was seen as a more a collaborative partnership with specific focus on (a) sharing of information (b) organizational participation, which is the formation of support structures such as health committees (c) co-operation by community members (d) involvement of the community in decision making about health programmes rendered at the clinic (e) communication between health professionals and the community members and ( $f$ ) contribution of skills in health care provision. In their own words: "It means being informed about health programmes being run in the clinic. It also means that people should have a say in how they should be treated in the clinic". (Rural community)

"Community involvement in health does not exist in this place but we are also part of the problem since we do not have proper channels of communication such as health committees where we can report health problems". (Rural community)

"My understanding of community involvement in health is that it refers to community's involvement in health committees". (Rural community)

Both urban and rural communities, understanding of $\mathrm{CIH}$ tallied very well with the characteristics of community participation as identified by different authors in the field of CIH (Dennill, King \& Swanepoel, 1999: 85; Kahssay \& Oakley, 1999: 7; Rifkin, Muller \& Bichmann, 1988: 932). Furthermore, according to the WHO (1985:31), access to information as well as the right of the people to exercise power over decisions that affect their lives are key characteristics of $\mathrm{CIH}$. The broad understanding of $\mathrm{CIH}$ by the rural community members might have been strengthened by the fact that these community members had been previously involved in development committees and health committees that existed in the community before the new municipality demarcations were put in place. This understanding of $\mathrm{CIH}$ means 


\begin{tabular}{|c|c|c|}
\hline Participants & Case A (Urban) & Case B (Rural) \\
\hline Community Members & $\begin{array}{l}\text { Collaboration: community } \\
\text { and health professionals have } \\
\text { to help each other in the } \\
\text { provision of health care }\end{array}$ & $\begin{array}{l}\text { Information: being informed about service provision and health } \\
\text { programmes. Collaboration: between the health professionals } \\
\text { and community. Establishment of health committees working } \\
\text { together in provision of health care. Involvement in decision- } \\
\text { making: involvement of community in decision making } \\
\text { Organization participation: the involvement of community in } \\
\text { health committees }\end{array}$ \\
\hline Health Professionals & $\begin{array}{l}\text { Involvement in decision- } \\
\text { making: community has to be } \\
\text { involved in decision making } \\
\text { about their needs' } \\
\text { identification. } \\
\text { Communication: the } \\
\text { community has to communi- } \\
\text { cate its needs to the health } \\
\text { professionals. The health } \\
\text { professionals should com- } \\
\text { municate with the community } \\
\text { by giving them advice. } \\
\text { Contributive participation: } \\
\text { both the community and the } \\
\text { health professionals should } \\
\text { contribute in health care } \\
\text { provision. }\end{array}$ & $\begin{array}{l}\text { Cooperation: community members to cooperate with the service } \\
\text { providers by attending clinic functions and utilizing the service. } \\
\text { Knowledge of community needs: the community needs to report } \\
\text { their health needs to the health professionals }\end{array}$ \\
\hline Community Health Workers & $\begin{array}{l}\text { Cooperation: the community } \\
\text { has to accept the health } \\
\text { programmes to show their } \\
\text { involvement. Collaboration: } \\
\text { the community and the health } \\
\text { professionals have to work } \\
\text { hand in hand during health } \\
\text { care provision. }\end{array}$ & $\begin{array}{l}\text { Cooperation: community members cooperating with service } \\
\text { providers during service provision, such as "choosing what } \\
\text { you eat when you have diabetes and hypertension and the } \\
\text { importance of taking medications". Collaboration: between } \\
\text { the community and the clinic. The community assisting clinic } \\
\text { staff should they need assistance, "we work together". }\end{array}$ \\
\hline
\end{tabular}

that health professionals have a good starting point in involving the community in health matters because the community knew exactly what they wanted in order to involve themselves in health matters and hence improve their health status. In the urban community, the community members who participated in the study had a very limited understanding of $\mathrm{CIH}$. This was not surprising because the participants were people who only visited the clinic occasionally, when there was a need. Their only understanding of CIH was that it meant mutual assistance and participating in health committees. This limited understanding of $\mathrm{CIH}$ could have been linked to the fact that these community members had never had any exposure to community development programmes, unlike the community members in the rural community. They came to the clinic only for treatment and therefore could not have been aware of the then existing health committees. This in turn might be attributed to the nature of relationships in urban communities. According to Mann's (1983: 409), relationships in urban communities tend to be impersonal, superficial and segmented.

The conceptualisation of $\mathrm{ClH}$ as collaboration by the rural community and as mutual assistance by the urban community should be seen as a positive finding; after all, the WHO (1985: 32) study group emphasized the necessity for collaboration with community representatives and where possible with community members. This view might be indicative of communities who are ready to work with health professionals in health care provision. Contrary to this finding, only the urban-based health professionals saw CIH as collaboration (community involvement in decision making and needs identification). Even then, it appeared as though this understanding was not shared with the community served, in that the urban community itself had seen $\mathrm{CIH}$ as merely mutual assistance rather collaborative participation in the provision of health care. This discrepancy poses a threat in both rural and urban communities since health professionals and community members need to work as partners to ensure effectiveness of the service rendered

\section{Health Professionals' Understanding of $\mathrm{ClH}$}

There was no common link between the urban and rural health professionals' understanding of $\mathrm{CIH}$. As can be seen in Table 2, for the urban health professionals. CIH meant (a) involvement 
of community members in decision making, (b) communication, and (c) contributive participation. For the rural health professionals, however, $\mathrm{CIH}$ meant cooperation and knowledge of community needs. The following are excerpts from the participants' statements.

"It means involvement of the people in decision making, that people should be involved in identifying their health needs and the running of the clinic". (urban)

"It means that community members should communicate their health needs to us and that needs to be taken into consideration". (urban)

"It means that the people should contribute more than you (health professional) because you have to develop them (community). It means that we as health workers have to empower the people through developing their skills". (urban)

"It means working with the community" as we are working in this clinic. The community takes part in health programmes, like when we have health days we always ask them to come and assist like giving speeches to the community: Unfortunately there is not much that they can do in assisting with the health programme. They can only help with speeches and with refreshments especially because we cannot all participate in the same things. We as nurses have to continue with our work at the same time". (rural)

"We need to know' their needs. We are here because of them. We also need to communicate with them to find out what their needs are". (rural)

Although the health professionals in the rural community mainly understood $\mathrm{CIH}$ to be the cooperation of the community members with the existing health programmes, for the community they served, CIH meant involvement in decision-making (see Table 2). This is in agreement with such authors as Kahssay and Oakley (1999: 9); Stanhope and Lancaster (1996: 45), that people's involvement should not just be in the support and functioning of health services but more importantly involvement in decision making and actions that affect their health in order to encourage a sense of responsibility. A similar view was supported by the WHO (1988:28) in stating that communities were seldom involved in the development of health programmes beyond "being expected to bring their children for immunization".

For the health professionals in the urban communities, however, CIH meant communication between the community members and the health professionals, involvement of community members in decision-making and contribution of both parties to the rendering of health care (see Table 2). This was seen to be a positive finding since the WHO (1985:35) identified the need that community residents and health providers needed to work in partnership as each had their area and level of expertise. Similarly, Stanhope and Lancaster (1996: 239) asserted that community members must participate in decisions about health in order to promote development and selfreliance. This partnership is needed in seeking solutions to the complex problems facing communities.

\section{Community Health Workers' Understanding of $\mathrm{ClH}$}

Themes emerging from both the urban and rural Community Health Workers' descriptions of their understanding of $\mathrm{CIH}$ were cooperation and collaboration. It means improving the level of health by working hand in hand with health workers. (Urban)

We have to be involved if there's something taking place at the clinic. It means assisting clinic staff as community members. (Rural)

The common understanding of $\mathrm{CIH}$ by the rural and urban community health workers can be attributed to the nature of their role in the communities. Part of the community health workers' work among other things, involves follow-up visits on those community members with chronic conditions, social support, advocating for individual and community needs, building individual and community capacity (Rosenthal, 1998, cited in Swider, 2002: 12). This means that if people are compliant in taking their treatment, then the CHWs saw them as being cooperative with the health programmes.

\section{Conclusions and \\ recommendations}

The researchers set out to explore the meaning of $\mathrm{CIH}$ for PHC communities in rural and urban health centres. It was expected that the two communities (rural and urban) would differ in their understanding of $\mathrm{CIH}$, if only because of the contexts in which $\mathrm{CIH}$ has to be operationalised. This expectation was supported by the findings of this study. Not only did the understanding of $\mathrm{CIH}$ differ between these two communities, but also between different participants within communities. When differences in understanding exist, it unlikely that the parties involved would be working toward a common goal.

Commonalities in understanding did, however, also exist. In both settings, for the community members who participated in this study, $\mathrm{CIH}$ means working with the health professionals whether by merely assisting each other or through collaboration between health professionals and the community. According to the rural community and the urban health professionals, such collaboration is more than cooperation with each other but must include involvement in decision-making, planning and implementation of health programmes.

Based on these results, it can be concluded that, although there are differences in understanding of $\mathrm{CIH}$ between and within groups, some commonalities exist between the rural and the urban communities' understanding and to a certain extent within communities.

It is essential, however, that the health professionals and the community they serve talk to each other, and ensure that they have a common understanding of $\mathrm{CIH}$ and that differences in understanding are clarified. It is only then that implementation of $\mathrm{CIH}$ in its essence is possible. Meetings or workshops could be held where this issue of community involvement can be discussed in depth and each community can come to an understanding as to how they will approach his issue. Their understanding need not necessarily be the same as in other communities but should be in line with PHC principles.

\section{Limitations of the study}

- The community members in both communities could not differentiate between the CHWs and the health professionals. In their responses, some of the community members kept on referring to the CHWs when they meant the health professionals and vice versa.

- The other limitation was that in Case $A$, the community members from the suburban residential areas were not utilizing the clinic as expected. The majority of clients attending the clinic were from areas outside town. The people who were 
utilizing the clinic were only in town for employment but did not live in town.

- Owing to the sampling method used the sample size was too small and not fully representative of the communities in both cases, therefore the study cannot be generalised.

- With such a small sample size, it was difficult to achieve information redundancy, which is one of the disadvantages of using purposive sampling (Burns \& Grove, 2001: 376)

- In case B, some community meetings had to be cancelled because of bad weather and transport problems for the participants.

\section{References}

AFRICAN NATIONAL CONGRESS 1994: The National Health Plan. Johannesburg: ANC.

BURNS, N \& GROVE, S K 2001: The practice of nursing research: Conduct, critique \& utilisation, $4^{\text {th }}$ edition. Philadelphia: W B Saunders company

CHIMERA-DAN, G 1996: Community involvement in urban health programs. South Africa: International Thomson publishing.

CLARK, M J 1996: Nursing in the community. Stamford: Appleton \& Lange.

CRABTREE, B F \& MILLER, W L 1992: Doing qualitative research: Research methods for primary care volume 3 . Newbury Park: Sage Publications.

DELOUGHERY, G L 1998: Issues and Trends in Nursing. $3^{\text {rd }}$ edition. St. Louis: Mosby

DENNILL, K; KING, L \& SWANEPOEL , T 1999: Aspects of primary health care: community health in Southern Africa. South Africa: Oxford university press.

DEPARTMENT OF HEALTH 1997: White Paper for the Transformation of the Health system in South Africa. Government Notice (No. 17910). Pretoria http://www.polity.org.za/govdocs/ white_papers/health.html, accessed on 03/02/2005

GLASER, B G \& STRAUSS, A L 1967: The Discovery of Grounded Theory. New York: Aldine Publication Co.
GWELE, NS \& MAKHANYA, NJ 2001:

The Management and Leadership Skills Training Programme for Primary Health Care Coordinators in Region B-KZN: An Evaluation Study. Unpublished manuscript.

KAHSSAY, H M \& OAKLEY, P 1999: Community involvement in health development: A review of the concept and practice. Geneva: WHO.

MANN, M 1983: The Macmillan student encyclopaedia of sociology. London: Macmillan Press

POLIT, DF \& HUNGLER, BP 1995: Nursing Research, Principles and Methods. $6^{\text {th }}$ edition. Philadelphia: Lippincott

RIFKIN, S B; MULLER, F \& BICHMANN, W 1988: Primary health care: On measuring participation. Social Science and Medicine. 26 (9), 931-940.

SEAMANS, C H C 1987: Research Methods: Principles, Practice, and Theory for Nursing. $3^{\text {rd }}$ edition Norwalk: Appleton \& Lange.

STANHOPE, M S \& LANCASTER, J 1996: Community Health Nursing: Process and practice for promoting health. St. Louis: Mosby.

SWIDDER, S M 2002: Outcome effectiveness of community health workers: An integrative literature review. Public Health Nursing. 19 (1), 11-20.

VAN RENSBURG, H C J 2004: Health and health care in South Africa. $1^{\text {st }}$ edition. Pretoria: Van Schaik.

VILJOEN, R; HEUNIS, C; JANSE VAN RENSBURG, E; VAN RENSBURG, D; ENGELBRECHT, M; FOURIE, A; STEYN, F \& MATEBESI, Z 2000: The National Primary Health Care Facilities Survey 2000. Durban: Health Systems Trust.

WHO 1978: Primary health care: Report of the international conference on primary health care, Alma Ata, USSR, 612 September 1978. Geneva: WHO.

WHO 1985: Community-Based Education of health personnel: Report of WHO study group, 6-12 November 1985. Geneva: WHO.

WHO 1988: From Alma Ata to the Year
2000: Reflections at the Midpoint. Geneva: WHO.

WOELK, GB 1992: Cultural and Structural influences in the creation of and participation in community health programmes. Social Science and Medicine. 35 (4) 419-424.

YIN, R K 2003: Case study research: Design and Methods. Newbury Park: Sage. 